\title{
Efficient Fusion of Spectrum Sensing Information under Parameter Uncertainty and Impulsive Noise
}

\author{
Pedro H. C. Souza, Dayan A. Guimarães and Guilherme P. Aquino
}

\begin{abstract}
This paper addresses the performance of an efficient fusion scheme for cooperative spectrum sensing in the context of cognitive radio systems. The secondary users' decisions are transmitted to the fusion center at the same time and using the same carrier frequency, thus saving bandwidth and time resources of the report channel. The report channel state information and the receiver thermal noise variance are the main parameters used by the decision rule at the fusion center to determine whether the primary signal is present or absent. The global spectrum sensing performance is addressed in this paper when the fusion center receiver is subjected to impulsive noise and uncertainty in the estimation of the above parameters. It is demonstrated that the receiver is quite robust against noise variance uncertainty and impulsive noise, whereas its performance may be severely degraded due to channel state information uncertainty.
\end{abstract}

Index Terms-Cognitive radio, fusion of spectrum sensing information, impulsive noise, channel state information, noise uncertainty.

\section{INTRODUCTION}

The world is currently witnessing an unparalleled increase in the interest for new wireless communication products and services. However, the current fixed radio-frequency allocation policy has been proved inefficient to cope with this demand, in spite of the spectrum underutilization in some regions and bands [1], [2]. Thus, new paradigms for the creation of other allocation and access strategies are needed.

The cognitive radio (CR) concept [3], [4] has come to tackle the inefficiency of the fixed allocation policy by allowing, among other features, the opportunistic access of CRs belonging to a secondary network to vacant primary network bands. CR networks are expected to drive the fifth generation (5G) of digital communications [5], which is why the CR topic nowadays is attracting a tremendous amount of interest from the academia and industry.

Spectrum sensing [6] is the CR feature that allows for detecting vacant bands (the so-called spectrum holes or whitespaces) in the primary network spectrum. The performance of the spectrum sensing is often measured in terms of the probability of detection and the probability of false alarm. The former is the probability of declaring a band occupied when the primary network is indeed using it. The later is the probability of declaring a band occupied when it is in fact vacant. A high probability of detection is desired to protect the primary network from harmful interference caused by the

Pedro H. C. Souza, Dayan A. Guimarães and Guilherme P. Aquino was with the National Institute of Telecommunications (Instituto Nacional de Telecomunicações - Inatel) - Brazil. e-mail: phcs0@hotmail.com, dayan@inatel.br and guilhermeaquino@inatel.br

Digital Object Identifier: 10.14209/jcis.2018.5 secondary users transmitting over a busy channel. On the other hand, a low false alarm probability is desired so that the opportunistic transmissions of the secondary network occur efficiently, increasing the network throughput [6]. However, these probabilities are conflicting metrics, meaning that the improvement of one is always accompanied by a degradation of the other. Thus, a trade-off is the solution.

Each CR, hereafter also referred to as secondary user (SU), may seek for a vacant band independently of the other SUs, but this approach is highly sensitive to channel fading and shadowing, yet being prone to the hidden terminal problem. The cooperative spectrum sensing (CSS) can cope with these problems by means of the spatial diversity produced by multiple SUs simultaneously monitoring the primary user (PU) signal activity [6].

In centralized CSS with data fusion [6], the signal samples collected by each $\mathrm{SU}$, or some other soft information derived from them, are sent to the fusion center (FC) over the report control channels, which typically have low bandwidth. From this information, the test statistic is then formed at the FC, where the final global decision on the spectrum occupancy is made. In centralized CSS with decision fusion [6], the SUs make their own local hard decisions on the spectrum occupancy and transmit them to the FC, where they are combined to feed the global decision process.

Although the use of soft information can yield more reliable decisions, it suffers from a high expenditure of report channel resources, since this information is typically sent through channels orthogonal in time, frequency, code, or some combination of these. This problem is aggravated when the number of SUs in cooperation increases [17]. The centralized CSS with decision fusion is preferred when the savings of report channel resources are of concern, since in principle a single bit per SU is needed to convey the spectrum sensing information to the FC [6]. Nevertheless, the report channel occupancy still grows with the increase of the number of SUs in cooperation.

Several research efforts have been spent to save resources during the report phase of the CSS process; see for instance [7]-[17] and references therein.

This paper is grounded on the proposal described in [17], where the author introduces a novel CSS scheme in which the SUs in cooperation simultaneously report their local decisions via binary phase-shift keying (BPSK) [32] symbols using the same carrier frequency, thus saving communication resources of the report channel. During the report phase, the received signal at the FC is formed by the non-coherent sum of the signals carrying all local SUs' decisions. The global decision rule provided in [17] operates under the assumption that the 
noise variance at the $\mathrm{FC}$ receiver input and the report channel gains between the SUs and the FC are known to the FC, so that each received signal sample can be classified according to the hypotheses of absence or presence of the PU signal. This classification applies the $K$-out-of- $M$ logic combining rule [6], [18], in which the FC decides in favor or the presence of the PU signal if $K$ or more SUs decide accordingly, where $M$ is the number of SUs in cooperation.

A modified maximum a posteriori (MAP) decision rule for the technique proposed in [17] is given in [19]. This rule yields performances by far superior to those achieved with the original rule of [17], whilst keeping approximately the same complexity.

Based on a simplified version of the decision rule given in [19], in [20] it is proposed a receiver structure which is capable of adapting its operation to the variations of the instantaneous signal-to-noise ratio (SNR) at the FC.

To the best of the authors' knowledge, none of the publications related to the technique proposed in [17] deal with the influence of uncertainty in the values of the noise variance at the FC receiver input and of the report channel gains (or channel state information) on the global spectrum sensing performance. Motivated by this fact, this paper addresses the global spectrum sensing performance of the receiver developed in [20] when it is subjected to uncertainty in the above parameters, which is a common situation in practice [21]-[27]. Moreover, motivated by the fact that digital communication receivers in general are sensitive to impulsive noise [28], [29], and that the performance of receivers for spectrum sensing applications may also be severely degraded in the presence of such impairment [30], this paper also assess the performance of the FC receiver of [20] under this impairment. To this end, the receiver was implemented on the solidThinking Embed/Comm (former VisSim/Comm) software platform version 8.0, which is a tool for modeling and simulating dynamical systems [31], [32]. In the corresponding Monte Carlo simulations of the CSS, the channel state information uncertainty was modeled according to [21]-[23], the noise variance uncertainty was modeled according to [25]-[27] and the compound (thermal plus impulsive) noise was simulated based on the symmetric alpha stable $(\mathrm{S} \alpha \mathrm{S})$ model [28], [29].

The remainder of the paper is organized as follows: Section II describes the resource-efficient CSS system model and corresponding decision rules. In Section III, the mathematical model of the receiver at the FC is presented. Section IV is devoted to the models for the channel state information and the noise variance uncertainties, as well as for the generation of the compound noise at the FC receiver input. Numerical results and discussions are given in Section V. Section VI concludes the paper.

Throughout the text, italicized lowercase letters (e.g. $x$ ) represent scalars, boldfaced lowercase letters (e.g. x) represent vectors, and boldfaced uppercase letters (e.g. X) denote matrices. The $n$-th element of the vector $\mathbf{x}$ is represented by $x_{n}$. The element on the $n$-th row and $m$-th column of the matrix $\mathbf{X}$ is denoted by $X_{n, m}$. The sets of real and complex numbers are respectively represented by $\mathbb{R}$ and $\mathbb{C}$. The absolute value of the scalar $x \in \mathbb{R}$ or the modulo of $x \in \mathbb{C}$ is denoted by $|x|$. The sets of matrices of order $x \times y$ with real and complex elements are respectively represented by $\mathbb{R}^{x \times y}$ and $\mathbb{C}^{x \times y}$. The transposition of the vector $\mathbf{x}$ and the matrix $\mathbf{X}$ are respectively represented as $\mathbf{x}^{T}$ and $\mathbf{X}^{\mathrm{T}}$. The superscript ${ }^{(\mathrm{T})}$ denotes a target performance metric. The Euclidean norm of the vector $\mathbf{x}$ is represented by $\|\mathbf{x}\|=\sqrt{\mathbf{x}^{\mathrm{T}} \mathbf{x}}$. The expected value of $z$ is denoted by $\mathbb{E}\{z\}$. The real and imaginary parts of $r \in \mathbb{C}$ are respectively denoted by $\Re(r)$ and $\Im(r)$. The operation $\operatorname{diag}(\mathbf{X})$ creates a vector formed by the elements on the main diagonal of the matrix $\mathbf{X}$. The estimate of a scalar $x$, a vector $\mathbf{x}$ or a matrix $\mathbf{X}$ is represented by $\hat{x}, \hat{\mathbf{x}}$ and $\hat{\mathbf{X}}$, respectively. The operation $\lfloor x\rfloor$ gives the largest integer not exceeding the scalar $x$. In complex numbers, $j=\sqrt{-1}$ is the imaginary unit.

\section{RESOURCE-EFFICIENT CSS MODEL}

In the CSS scheme of [17], $M$ secondary users simultaneously transmit their local hard decisions to the FC using BPSK modulation, with the same carrier frequency. Let $m_{k}$ represent the binary local spectrum sensing decision made by the $k$-th $\mathrm{SU}, k=1, \ldots, M$, with $m_{k}=1$ indicating the presence of the PU signal (hypothesis $H_{1}$ ) and $m_{k}=0$ indicating no active PU (hypothesis $H_{0}$ ). The baseband equivalent of the BPSK symbol with energy $E_{\mathrm{b}}$ transmitted by the $k$-th $\mathrm{SU}$ is

$$
s_{k}=\left(2 m_{k}-1\right) \sqrt{E_{\mathrm{b}}} .
$$

The complex received signal sample at the FC in a given arbitrary sensing round is given by

$$
r=\sum_{k=1}^{M} h_{k} s_{k}+w,
$$

where $h_{k} \in \mathbb{C}$ denotes the gain of the reporting channel between the $k$-th $\mathrm{SU}$ and the $\mathrm{FC}$, and $w \in \mathbb{C}$ is the thermal zero-mean additive white Gaussian noise (AWGN) sample with variance $\sigma_{w}^{2}$ volts $^{2}$ (or watts), and with power spectral density $N_{0}=2 \sigma_{w}^{2}$ watts/hertz.

The noiseless received symbols, which from (2) are given by $\sum_{k=1}^{M} h_{k} s_{k}$, can take one of $2^{M}$ possible values and can be seen as the weighted sum of independent and identically distributed (i.i.d.) Bernoulli random variables [19]. The exact probabilities of these noiseless symbols can be obtained from equation (13) of [33] as

$$
P_{i}(p)=\prod_{k=1}^{M}(1-p)^{1-S_{k, i}} p^{S_{k, i}}, \quad i=1, \ldots, 2^{M},
$$

where $p=P_{\mathrm{D}, \mathrm{SU}}$ or $p=P_{\mathrm{FA}, \mathrm{SU}}$, with $P_{\mathrm{D}, \mathrm{SU}}$ and $P_{\mathrm{FA}, \mathrm{SU}}$ being, respectively, the probability of detection and the probability of false alarm at each SU terminal, and $S_{k, i} \in\{0,1\}$ are the elements of the matrix $\mathbf{S}^{M \times 2^{M}}$ whose columns are formed by all possible $M$-element sets of SUs' decisions.

Let the secondary users' decisions be arranged in the vector $\mathbf{s}=\left[s_{1}, s_{2}, \ldots, s_{M}\right]^{\mathrm{T}}$, and the report channel gains arranged in the vector $\mathbf{h}=\left[h_{1}, h_{2}, \ldots, h_{M}\right]^{\mathrm{T}}$. Additionally, let $D_{1}$ and $D_{0}$ represent the sets of local decision vectors 
associated to the choice of $H_{1}$ and $H_{0}$, respectively, on the basis of the $K$-out-of- $M$ rule, that is,

$$
\begin{aligned}
& D_{1}=\left\{\mathbf{s} \mid \sum_{k=1}^{M} m_{k} \geq K\right\}, \\
& D_{0}=\left\{\mathbf{s} \mid \sum_{k=1}^{M} m_{k}<K\right\},
\end{aligned}
$$

in which the numbers of elements are respectively [19]

$$
\begin{aligned}
\mathcal{L}_{1} & =\sum_{i=K}^{M}\left(\begin{array}{c}
M \\
i
\end{array}\right), \\
\mathcal{L}_{0} & =2^{M}-\mathcal{L}_{1} .
\end{aligned}
$$

According to the decision rule proposed in [17], the FC decides in favor of $H_{1}$ if

$$
\sum_{\mathbf{s} \in D_{1}} \exp \left(-\frac{\left|r-\mathbf{h}^{\mathrm{T}} \mathbf{s}\right|^{2}}{2 \sigma_{w}^{2}}\right) \geq \sum_{\mathbf{s} \in D_{0}} \exp \left(-\frac{\left|r-\mathbf{h}^{\mathrm{T}} \mathbf{s}\right|^{2}}{2 \sigma_{w}^{2}}\right)
$$

and decides in favor of $H_{0}$ otherwise.

In the modified MAP decision rule proposed in [19], the FC decides in favor of $H_{1}$ if

$$
\sum_{\mathbf{s} \in D_{1}} P_{\mathbf{s}} \exp \left(-\frac{\left|r-\mathbf{h}^{\mathrm{T}} \mathbf{s}\right|^{2}}{2 \sigma_{w}^{2}}\right) \geq \sum_{\mathbf{s} \in D_{0}} P_{\mathbf{s}} \exp \left(-\frac{\left|r-\mathbf{h}^{\mathrm{T}} \mathbf{s}\right|^{2}}{2 \sigma_{w}^{2}}\right)
$$

and chooses $H_{0}$ otherwise. In this rule, the uniform prior probability of symbol s implicitly assumed in both sides of (6) is made explicit, and is given by

$$
P_{\mathbf{s}}=P_{H_{1}} P_{i}\left(P_{\mathrm{D}, \mathrm{SU}}^{(\mathrm{T})}\right)+P_{H_{0}} P_{i}\left(P_{\mathrm{FA}, \mathrm{SU}}^{(\mathrm{T})}\right),
$$

where $P_{H_{0}}$ and $P_{H_{1}}$ are the prior probabilities of the hypotheses $H_{0}$ and $H_{1}$, which are assumed to be the same to represent the maximum entropy on the PU activity state, and $P_{i}(p)$ is from (3) for $i: \mathbf{s} \in D_{0}$ or $D_{1}$, with $p=P_{\mathrm{D}, \mathrm{SU}}^{(\mathrm{T})}$ or $p=P_{\mathrm{FA}, \mathrm{SU}}^{(\mathrm{T})}$ being the target probabilities of detection and false alarm achieved by the SUs. These probabilities are computed from the corresponding target probabilities $P_{\mathrm{D}, \mathrm{FC}}^{(\mathrm{T})}$ and $P_{\mathrm{FA}, \mathrm{FC}}^{(\mathrm{T})}$ at the FC in the error-free situation [34], inverting

$$
\begin{aligned}
P_{\mathrm{D}, \mathrm{FC}}^{(\mathrm{T})} & =\sum_{i=K}^{M}\left(\begin{array}{c}
M \\
i
\end{array}\right) P_{\mathrm{D}, \mathrm{SU}}^{(\mathrm{T})}{ }^{i}\left(1-P_{\mathrm{D}, \mathrm{SU}}^{(\mathrm{T})}\right)^{M-i}, \\
P_{\mathrm{FA}, \mathrm{FC}}^{(\mathrm{T})} & =\sum_{i=K}^{M}\left(\begin{array}{c}
M \\
i
\end{array}\right) P_{\mathrm{FA}, \mathrm{SU}}^{(\mathrm{T})}{ }^{i}\left(1-P_{\mathrm{FA}, \mathrm{SU}}^{(\mathrm{T})}\right)^{M-i} .
\end{aligned}
$$

The target probabilities of detection and false alarm at the FC represent to the global spectrum sensing performance and in practice can be set to comply with standardized specifications like those in the IEEE 802.22 [35], which is the first released standard on the cognitive radio technology.

\section{Adaptive Receiver Model}

The decision rules (6) and (7) bring little or no insight about their implementations in real receivers, except if one think of realizing all operations as they natively are. In [36] and [20], an approximation of the logarithm of the sum of exponentials (log-sum-exp [37, p. 72]) has been adapted to the structure of a quadrature receiver, so that the implementation of the decision rule at the FC becomes simpler. The reasoning goes as follows. Admit that the FC received signal, $r(t)$, is processed by a quadrature receiver [32] that generates a vector containing the real and imaginary parts of $r$ according to

$$
\mathbf{r}=\left[\begin{array}{l}
\Re(r) \\
\Im(r)
\end{array}\right]=\left[\begin{array}{l}
\int_{t}^{t+T_{\mathrm{s}}} r(t) \sqrt{\frac{2}{T_{\mathrm{s}}}} \cos \left(2 \pi f_{\mathrm{c}} t+\theta\right) d t \\
\int_{t}^{t+T_{\mathrm{s}}} r(t) \sqrt{\frac{2}{T_{\mathrm{s}}}} \operatorname{sen}\left(2 \pi f_{\mathrm{c}} t+\theta\right) d t
\end{array}\right],
$$

where $T_{\mathrm{s}}$ is the BPSK symbol duration, $f_{\mathrm{c}}$ is the report channel carrier frequency and $\theta$ is an unknown initial carrier phase. Also, assume that the matrices $\hat{\mathbf{L}}_{1} \in \mathbb{R}^{2 \times \mathcal{L}_{1}}$ and $\hat{\mathbf{L}}_{0} \in \mathbb{R}^{2 \times \mathcal{L}_{0}}$ are defined according to

$$
\hat{\mathbf{L}}_{1}=\left[\begin{array}{c}
\Re\left(\hat{\mathbf{v}}_{1}^{\mathrm{T}}\right) \\
\Im\left(\hat{\mathbf{v}}_{1}^{\mathrm{T}}\right)
\end{array}\right] ; \quad \hat{\mathbf{L}}_{0}=\left[\begin{array}{c}
\Re\left(\hat{\mathbf{v}}_{0}^{\mathrm{T}}\right) \\
\Im\left(\hat{\mathbf{v}}_{0}^{\mathrm{T}}\right)
\end{array}\right],
$$

where the elements of $\hat{\mathbf{v}}_{1} \in \mathbb{C}^{\mathcal{L}_{1} \times 1}$ and $\hat{\mathbf{v}}_{0} \in \mathbb{C}^{\mathcal{L}_{0} \times 1}$ are given by $\hat{\mathbf{h}}^{\mathrm{T}} \mathbf{s}$, respectively for $\mathbf{s} \in D_{1}$ and $\mathbf{s} \in D_{0}$.

Knowing that $|x|^{2}=x x^{*}$ for $x \in \mathbb{C}$, it can be easily verified that $\left|r-\mathbf{h}^{\mathrm{T}} \mathbf{s}\right|=\left[\Re(r)-\Re\left(\mathbf{h}^{\mathrm{T}} \mathbf{s}\right)\right]^{2}+\left[\Im(r)-\Im\left(\mathbf{h}^{\mathrm{T}} \mathbf{s}\right)\right]^{2}$. Then, denoting $\mathbf{L}_{1 \mathrm{~s}}$ as the column of $\mathbf{L}_{1}$ associated to the symbol $\mathbf{s} \in D_{1}$, we can write that $\left|r-\mathbf{h}^{\mathrm{T}} \mathbf{s}\right|=\left\|r-\mathbf{L}_{1 \mathbf{s}}\right\|^{2}$ and, by analogy, $\left|r-\mathbf{h}^{\mathrm{T}} \mathbf{s}\right|=\left\|r-\mathbf{L}_{0 \mathbf{s}}\right\|^{2}$ for $\mathbf{s} \in D_{0}$.

For high values of $E_{\mathrm{b}} / N_{0}$ in the report channels, the logarithm can be applied to both sides of (7) using the lower bound of $\max \left(a_{1}, . ., a_{\mathcal{L}}\right) \leq \ln \sum_{i=1}^{\mathcal{L}} \exp \left(a_{i}\right) \leq \max \left(a_{1}, . ., a_{\mathcal{L}}\right)+\ln \mathcal{L}$ as an approximation of $\ln \sum_{i=1}^{\mathcal{L}} \exp \left(a_{i}\right)$ [37, p. 72]. On the other hand, for low values of $E_{\mathrm{b}} / N_{0}$, the approximation $\ln \sum_{i=1}^{\mathcal{L}} \exp \left(a_{i}\right) \approx \max \left(a_{1}, \ldots, a_{\mathcal{L}}\right)+k \ln \mathcal{L}$ for $k \leq 1$ is more tight and it is used instead. Therefore, applying these approximations and the results from the previous paragraphs in the left side of (7), it follows that

$$
\begin{aligned}
\ln \sum_{\mathbf{s} \in D_{1}} & P_{\mathbf{s}} \exp \left(-\frac{\left\|\mathbf{r}-\mathbf{L}_{1 s}\right\|^{2}}{2 \sigma_{w}^{2}}\right) \approx \\
& \max _{\mathbf{s} \in D_{1}}\left\{2 \sigma_{w}^{2} \ln P_{\mathbf{s}}-\left\|\mathbf{r}-\mathbf{L}_{1 \mathbf{s}}\right\|^{2}\right\}+k \ln \mathcal{L}_{1},
\end{aligned}
$$

where the argument of the max function can be written as $2 \sigma_{w}^{2} \ln P_{\mathbf{s}}-\left\|\mathbf{r}-\mathbf{L}_{1 \mathbf{s}}\right\|^{2}=2 \sigma_{w}^{2} \ln P_{\mathbf{s}}+2 \mathbf{r}^{\mathrm{T}} \mathbf{L}_{1 \mathbf{s}}-\mathbf{L}_{1 \mathbf{s}}^{\mathrm{T}} \mathbf{L}_{1 \mathbf{s}}-\mathbf{r}^{\mathrm{T}} \mathbf{r}$.

Applying the above approximations to the right-hand part of (7), an expression analogous to (12) is obtained. This development was omitted here for the sake of conciseness.

Hence, after the approximations are applied to both sides of (7) the adaptive decision rule is found. In this rule, the FC decides in favor of $H_{1}$ if

$$
\begin{aligned}
& \max \left\{2 \hat{\sigma}_{w}^{2} \ln \mathbf{p}_{1}+2 \hat{\mathbf{L}}_{1}^{\mathrm{T}} \mathbf{r}-\operatorname{diag}\left(\hat{\mathbf{L}}_{1}^{\mathrm{T}} \hat{\mathbf{L}}_{1}\right)\right\}+\hat{\sigma}_{w}^{2} \phi \ln \mathcal{L}_{1} \geq \\
& \max \left\{2 \hat{\sigma}_{w}^{2} \ln \mathbf{p}_{0}+2 \hat{\mathbf{L}}_{0}^{\mathrm{T}} \mathbf{r}-\operatorname{diag}\left(\hat{\mathbf{L}}_{0}^{\mathrm{T}} \hat{\mathbf{L}}_{0}\right)\right\}+\hat{\sigma}_{w}^{2} \phi \ln \mathcal{L}_{0},
\end{aligned}
$$

and decides in favor of $H_{0}$ otherwise. In this expression, $\mathbf{p}_{1} \in$ $\mathbb{R}^{\mathcal{L}_{1} \times 1}$ and $\mathbf{p}_{0} \in \mathbb{R}^{\mathcal{L}_{0} \times 1}$, with $\mathcal{L}_{1}$ and $\mathcal{L}_{0}$ given by (5), are the vectors whose elements are the probabilities $P_{\mathbf{s}}$ given in (8), respectively for $\mathbf{s} \in D_{1}$ and $\mathbf{s} \in D_{0}$.

Still referring to (13), the variable $\phi \in\{1,0\}$ is responsible for controlling the adaptation of the receiver to the instantaneous SNR. This adaptation works by adding (making $\phi=1)$ the terms $\hat{\sigma}_{w}^{2} \ln \mathcal{L}_{1}$ and $\hat{\sigma}_{w}^{2} \ln \mathcal{L}_{0}$ to the left-hand and 
the right-hand sides of the inequality, respectively, whenever the instantaneous $\operatorname{SNR}\left(\sigma_{s}^{2} / \hat{\sigma}_{w}^{2}\right)\|\hat{\mathbf{h}}\|^{2}$ is below a specified threshold. The role of the above-mentioned terms is to consider both bounds of the log-sum-exp approximation to the $\max$ function [37, p. 72], one suitable to high instantaneous SNRs and the other more adequate to low instantaneous SNRs [20].

Notice that the instantaneous SNR is assumed to be computed from the known transmitted signal power $\sigma_{s}^{2}$, and from the estimates of the noise variance and the channel gains. This is a quite pessimistic assumption, since this computation will yield in very inaccurate results when these estimates tend to their opposite limit values. In practice, an amelioration could be achieved if the instantaneous SNR is independently estimated by means of appropriate techniques [38], thus avoiding cumulative errors.

Finally, observe from (13) that the estimate $\hat{\sigma}_{w}^{2}$ of the noise variance, the estimate $\hat{\mathbf{h}}=\left[\hat{h}_{1}, \hat{h}_{2}, \cdots, \hat{h}_{M}\right]^{\mathrm{T}}$ of the channel gains implicit in $\hat{\mathbf{L}}_{1}$ and $\hat{\mathbf{L}}_{0}$, and the type of noise implicit in $\mathbf{r}$ do influence the global spectrum sensing performance, since the global decision rule makes use of these estimates.

\section{UnCERTAINTIES AND IMPULSIVE NOISE MODELS}

The models for uncertainties in the report control channel gains and thermal noise variance estimates are presented in this section, along with the model for generating the compound (thermal plus impulsive) noise at the input of the FC receiver.

\section{A. Uncertainty of the report channel gain estimate}

Based on the model given in [21], which is also adopted in [22]-[24], the estimate of the complex report channel gain between the $k$-th $\mathrm{SU}$ and the FC can be modeled as

$$
\hat{h}_{k}=h_{k} \rho+\epsilon_{k} \sqrt{1-\rho^{2}},
$$

where $h_{k}$ is the actual complex channel gain, $0 \leq \rho \leq 1$ is the magnitude of the normalized complex correlation between $\hat{h}_{k}$ and $h_{k}$, and $\epsilon_{k}$ is a zero-mean complex Gaussian random variable with variance $\sigma_{\epsilon}^{2}$ and independent of $h_{k}$ [22]. In this model, $\rho$ controls de degree of uncertainty of the channel estimate. If $\rho=1$, a perfect estimate is modeled, i.e. $\hat{h}_{k}=h_{k}$. As $\rho$ decreases towards zero, more uncertainty is modeled. When $\rho=0$, the estimate is a complex Gaussian variable completely decorrelated from $h_{k}$.

The manner in which the weights $\rho$ and $\sqrt{1-\rho^{2}}$ are used in (14) guarantees that the mean-square values of $\hat{h}_{k}$ and $h_{k}$ are the same for any $\rho$, that is $\mathbb{E}\left\{\left|\hat{h}_{k}\right|^{2}\right\}=\mathbb{E}\left\{\left|h_{k}\right|^{2}\right\}$, as long as $\sigma_{\epsilon}^{2}=\mathbb{E}\left\{\left|h_{k}\right|^{2}\right\}$. Since it is usual to consider that a fading channel has unitary power gain in terms of modeling, it follows that $\sigma_{\epsilon}^{2}=1$.

\section{B. Uncertainty of the thermal noise variance estimate}

Thermal noise uncertainty can be produced by the inherent inaccuracy of the noise variance estimation process, by the noise intensity itself (which depends on the receiver technology and temperature), as well as other impairments present at the receiver input.
A well-accepted model for thermal noise variance uncertainty considers that the variance lies in-between two extreme values, i.e.,

$$
\hat{\sigma}_{w}^{2} \in\left[\frac{\sigma_{w}^{2}}{\mu}, \mu \sigma_{w}^{2}\right],
$$

where $\mu \geq 1$ defines the lower limit $\sigma_{w}^{2} / \mu$ and the upper limit $\mu \sigma_{w}^{2}$ for the noise variance estimation error. It is common that the uncertainty in $\hat{\sigma}_{w}^{2}$ is expressed in $\mathrm{dB}$, that is, $\mu_{\mathrm{dB}}=$ $10 \log \mu[25]-[27]$.

There are two typical ways of generating the noise variance estimate under uncertainty, which are: i) $\hat{\sigma}_{w}^{2} \sim \mathcal{U}\left[\sigma_{w}^{2} / \mu, \mu \sigma_{w}^{2}\right]$, that is, $\hat{\sigma}_{w}^{2}$ is a random variable uniformly distributed in $\left[\sigma_{w}^{2} / \mu, \mu \sigma_{w}^{2}\right]$; ii) $\hat{\sigma}_{w}^{2}$ is generated with one of the extreme values and then with the other so that only worst-case scenarios are taken into account. In this paper we consider both ways.

\section{Impulsive noise}

The majority of communication systems analyses consider that the thermal noise (i.e., the AWGN) is the single noise impairment affecting the system performance. However, other noise types may severely degrade performance if they are not taken into account during the system development and configuration. The impulsive noise is one of these noise types [28]-[30].

Impulsive noise in communication systems can be generated from a variety of mechanisms, for instance: i) from the electrical mains or by direct induction on the receiver or ii) captured by the receiver antenna. In the first mechanism, the most common sources are the ignition system of ovens, the control system of dishwasher machines, thermostats of heaters, and switches of lamps. In the second mechanism, typical sources are lightning and the ignition system of cars [39].

Several models are available in the literature for characterizing the impulsive noise [28], [39]-[42]. Here we adopt the one discussed in [28], [42], in which the compound (thermal plus impulse) noise waveform is generated as a symmetric $\alpha$-stable $(\mathrm{S} \alpha \mathrm{S})$ random process whose probability density function $(\mathrm{PDF})$ is

$$
\psi(t)=\exp \left\{\left\lfloor j \delta t-\gamma|t|^{\alpha}\right\rfloor\right\} .
$$

The parameter $\alpha$ in (16), $0<\alpha \leq 2$ is the characteristic exponent that shapes the PDF. The lower the value of $\alpha$, the more heavy-tailed is the PDF, which is associated to stronger impulsive noise. For $\alpha=2$, the $\mathrm{S} \alpha \mathrm{S}$ distribution becomes Gaussian. The parameter $\delta$ is the mean of the distribution, which is set to zero to model a zero-mean compound noise. The parameter $\gamma>0$ characterizes the dispersion of the PDF, similarly to the concept of variance. The $\mathrm{S} \alpha \mathrm{S}$ distribution (as well as all distributions of the $\alpha$-stable family) has undefined variance for $\alpha<2$, and undefined mean for $\alpha \leq 1$ [28], [42].

The situation in which $\alpha=2$ is used as a reference to configure the average SNR per bit $E_{\mathrm{b}} / N_{0}$ at the input of the FC receiver when $\alpha \neq 2$, with $E_{\mathrm{b}}$ denoting the average energy per received bit and $N_{0}$ being the thermal noise power spectral density. In this situation $2 \gamma^{2}=\sigma_{w}^{2}$ [43], which allows for determining the scaling parameter as

$$
\gamma=\frac{\sigma_{w}}{\sqrt{2}}=\frac{\sqrt{N_{0}}}{2}
$$


Since $\sigma_{w}^{2}=N_{0} / 2$, it follows that the relation between $\gamma$ and the desired $E_{\mathrm{b}} / N_{0}$, in $\mathrm{dB}$, is given by

$$
\gamma=\sqrt{\frac{E_{\mathrm{b}}}{4 \times 10^{\frac{E_{\mathrm{b}} / N_{0}}{10}}}} .
$$

The above computation of $\gamma$ is exact only when $\alpha=2$, the unique situation in which the variance of the $S \alpha S$ process is finite. Then, the actual $E_{\mathrm{b}} / N_{0}$ for a given value of $\gamma$ is approximately equal to the desired value when $\alpha \neq 2$ [43].

\section{Numerical RESUlts}

This section is devoted to the analysis of the global spectrum sensing performance when the FC receiver is subjected to impulsive noise and to uncertainties in the thermal noise variance and in the report channel gain estimates.

\section{A. Performance metrics and simulation setup}

Two metrics were adopted to assess the system performance. One is the widely used receiver operating characteristic (ROC) curve, which trades the global probability of detection $P_{\mathrm{D}, \mathrm{FC}}$ as a function of the global probability of false alarm $P_{\mathrm{FA}, \mathrm{FC}}$. The other metric is the global probability of error, which is defined as

$$
P_{\mathrm{e}}=P_{H_{0}} P_{\mathrm{FA}, \mathrm{FC}}+P_{H_{1}}\left(1-P_{\mathrm{D}, \mathrm{FC}}\right) .
$$

The probability of error is useful for substituting or complementing the ROC curve, avoiding misinterpretations when, for instance, different ROC curves cross each other or the number of ROC results is large to the point of making the plots clumsy.

In this paper, only the minimum value of the probability of error for all pairs of $P_{\mathrm{FA}, \mathrm{FC}}$ and $P_{\mathrm{D}, \mathrm{FC}}$ of a given ROC curve is considered, for $P_{H_{0}}=P_{H_{1}}=1 / 2$. It can be easily shown that the point $\left(P_{\mathrm{FA}, \mathrm{FC}}, P_{\mathrm{D}, \mathrm{FC}}\right)$ that yields the minimum $P_{\mathrm{e}}$ of a ROC curve is approximately the point that is closest to the upper-left corner of the $P_{\mathrm{FA}, \mathrm{FC}}$ versus $P_{\mathrm{D}, \mathrm{FC}}$ graph, this proximity becoming tighter as $P_{\mathrm{FA}, \mathrm{FC}} \rightarrow 0$ and $P_{\mathrm{D}, \mathrm{FC}} \rightarrow 1$.

The CSS system with the FC receiver whose model is described in Section III was implemented on the the solidThinking Embed/Comm (former VisSim/Comm) software tool [31], [32], version 8.0, resulting in 913 native blocks of the tool. The reason for choosing this tool was that the implemented system closely resembles the operation of its actual continuous-time counterpart, thus facilitating system analysis and understanding. The resultant executable file is available at [44] for download.

Hereafter, each point on a ROC curve was generated from 100,000 runs (Monte Carlo events) of the simulation, each run corresponding to: i) the configuration of a decision threshold for the SUs; ii) the computation of the corresponding $P_{\mathrm{D}, \mathrm{SU}}$ and $P_{\mathrm{FA}, \mathrm{SU}}$, assuming for instance an energy detector (any other detector could be considered as well); iii) the generation of a pair of decision vectors $\mathbf{s}$ from a binomial distribution with parameters $M$ and $p=P_{\mathrm{D}, \mathrm{SU}}$ or $p=P_{\mathrm{FA}, \mathrm{SU}}$; iv) the modulation and simultaneous transmission of the SUs' decisions to the FC via BPSK modulation; v) the reception of the SUs' signals at the FC after having traveled through the report channels; and vi) the global decision at the FC based on the decision rule (13), under the combining rule $K$-ou-of- $M$ for $M=5$, and for $K=1, K=3$ and $K=5$. These values of $K$ respectively configure the well-known combining rules OR, majority voting (MAJ) and AND.

The local spectrum sensing at the SUs was simulated in such a way that $P_{\mathrm{D}, \mathrm{SU}}=P_{\mathrm{D}, \mathrm{SU}}^{(\mathrm{T})}$ and $P_{\mathrm{FA}, \mathrm{SU}}=P_{\mathrm{FA}, \mathrm{SU}}^{(\mathrm{T})}$ were necessarily generated for some value of the SUs' decision threshold. In each simulation run, a new and independent vector $\mathbf{h}$ was generated, with elements following a zero-mean complex Gaussian distribution with unitary second moment. This simulates flat and slow Rayleigh fading report control channels. For each pair of decision vectors $\mathbf{s}$, two received signal samples at the FC were generated according to (2). One signal sample was used to estimate $P_{\mathrm{FA}, \mathrm{FC}}$ and the other was used to estimate $P_{\mathrm{D}, \mathrm{FC}}$.

The probabilities $P_{\mathbf{s}}$ defined in (8) and used in the decision rule (7) were computed adopting $P_{\mathrm{FA}, \mathrm{FC}}^{(\mathrm{T})}=0.05$ and $P_{\mathrm{D}, \mathrm{FC}}^{(\mathrm{T})}=0.95$. These target performances comply with the IEEE 802.22 standard specifications, which are respectively $P_{\mathrm{FA}, \mathrm{FC}}^{(\mathrm{T})} \leq 0.1$ and $P_{\mathrm{D}, \mathrm{FC}}^{(\mathrm{T})} \geq 0.9[35]$.

The instantaneous SNR threshold that determines the operation of the adaptive receiver (see Section III) was set as $2 \mathrm{~dB}$ for $M=5$, using the same procedure described in [20].

\section{B. Performance under impulsive noise}

Figure 1 presents the global spectrum sensing performance in terms of ROC curves, as achieved by the adaptive receiver under impulsive noise with $\alpha=1.5,1.7$ and 2. Recall that $\alpha=1.5$ represents a strong impulsiveness in the compound noise, whereas $\alpha=2$ corresponds to a pure Gaussian noise. The values of $E_{\mathrm{b}} / N_{0}=3 \mathrm{~dB}$ and $11 \mathrm{~dB}$ at the input of the FC receiver were chosen to mimic a situation in which the system performance hits the target, and another situation in which the target is not reached at all. The number of SUs was $M=5$, with $K=1$ (OR), $K=3$ (MAJ) and $K=5$ (AND) in the $K$-out-of- $M$ rule. Perfect channel gain and noise variance estimates are considered in the results shown in this figure.

It can be noticed from Figure 1 that the MAJ rule outperforms the OR and the AND rules in terms of relative performance and immunity to impulsive noise. The performance variation under this impairment, from the situation of heavy impulsiveness $(\alpha=1.5)$ to no impulsiveness $(\alpha=2)$, is noticed to be small in the MAJ rule. The probability of detection practically does not change in the case of the OR rule, whereas the probability of false alarm changes significantly. The opposite behavior happens in the case of the AND rule. In all cases, the performance degrades as the signal-to-noise ratio per bit $E_{\mathrm{b}} / N_{0}$ decreases, as expected.

\section{Performance under thermal noise variance uncertainty}

Figure 2 shows the global spectrum sensing performance in terms of ROC curves for the adaptive receiver under noise variance uncertainties with $\mu_{\mathrm{dB}}=0,1,-1 \mathrm{~dB}$, and $\mu \sim \mathcal{U}\left[\sigma_{w}^{2} / \mu, \mu \sigma_{w}^{2}\right]=\mathcal{U}[1 / 1.259,1.259]$, for $E_{\mathrm{b}} / N_{0}=3 \mathrm{~dB}$ and $11 \mathrm{~dB}, M=5$, and $K=1,3$ and 5 . The extreme 

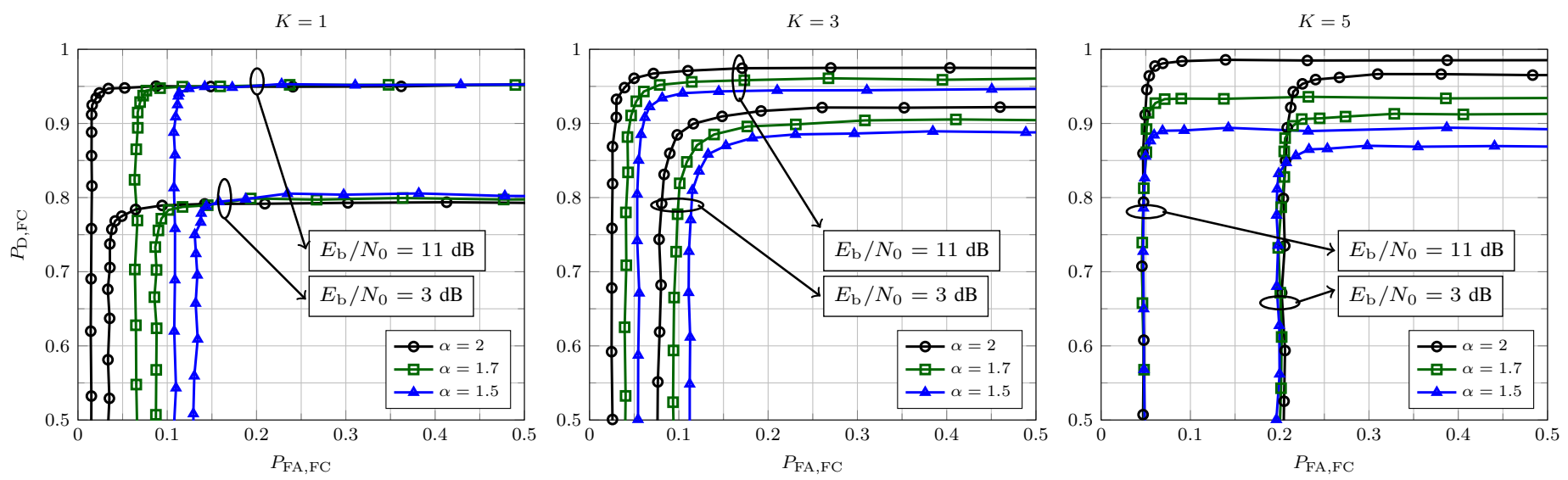

Fig. 1. Global spectrum sensing performance in terms of ROCs, as achieved by the adaptive receiver under impulsive noise with $\alpha=1.5,1.7$ and 2 , for $E_{\mathrm{b}} / N_{0}=3 \mathrm{~dB}$ and $11 \mathrm{~dB}, M=5$, and $K=1$ (left), $K=3$ (middle) and $K=5$ (right). This figure is better visualized in color.

noise uncertainties $\mu_{\mathrm{dB}}= \pm 1 \mathrm{~dB}$ have been chosen to represent a realistic, but relatively poor noise variance estimate [25]-[27]. Perfect channel estimates and no impulsive noise are considered in the results shown in Figure 2. It can be observed from this figure that the noise uncertainty does not produce large performance degradations in the global spectrum sensing performance, especially in the case of the MAJ rule, whose performance has been practically unaffected. This is particularly true for higher values of $E_{\mathrm{b}} / N_{0}$.

An interesting fact can be seen in Figure 2 when the estimated noise variance $\hat{\sigma}_{w}^{2}$ is made uniformly distributed around the actual value $\sigma_{w}^{2}$. Notice that the spectrum sensing performance under uniformly-distributed noise uncertainty lies very close to the one with no uncertainty, meaning that the amount of improvement brought by lowering the noise variance estimate with respect to the actual one is equal to the degradation brought by increasing the estimate in the same amount. This is not a common situation and means that actual unbiased estimators will tend to keep the performance of the spectrum sensing practically unchanged with respect to the perfect knowledge of the noise variance. Likewise observed in the case of impulsive noise contamination, the performance degrades as the signal-to-noise ratio per bit decreases, as expected, for all combining rules.

\section{Performance under report channel gain uncertainty}

Figure 3 gives the global spectrum sensing performance in terms of the probability of error $P_{\mathrm{e}}$ versus $E_{\mathrm{b}} / N_{0}$, as achieved by the adaptive receiver under uncertainty in the report channel gain with uncertainty parameter $\rho=1,0.99,0.97,0.95$ and 0.9 , for $M=5$, and $K=1,3$ and 5 . The use of $P_{\mathrm{e}}$ instead of ROC curves is now justified by the fact that the ROCs change their positions in a non-monotonic way as the channel uncertainty increases or decreases monotonically, making it difficult to analyze different performance results. Perfect noise variance estimates and no impulsive noise are considered in the results shown in this figure.

Figure 3 unveils two important behaviors: the first one is that the spectrum sensing performance is very sensitive to uncertainty in the report channel estimate. Notice that, with $\rho=0.99$, the target $P_{\mathrm{e}}=\frac{1}{2} 0.05+\frac{1}{2}(1-0.95)=0.05$ is barely achieved around $E_{\mathrm{b}} / N_{0}=13 \mathrm{~dB}$; the target $P_{\mathrm{e}}$ is not achieved for any other $\rho<0.99$. The AND and the OR rules have quite the same sensitivity to channel uncertainty, whereas the MAJ rule is a little more robust. The second important behavior is that $P_{\mathrm{e}}$ does not monotonically decreases when $E_{\mathrm{b}} / N_{0}$ is increased, as one would expect, except in the perfect channel estimation condition $(\rho=1)$. When the SNR per bit is low, the performance is poor (high $P_{\mathrm{e}}$ ), no matter the degree of correlation between the channel gain estimate and the actual channel gain, $\rho$, due to the dominance of the thermal noise over $\rho$. When $E_{\mathrm{b}} / N_{0}$ is high, the degree of correlation $\rho$ seems to dominate, yielding good performances for high values of $\rho$ and poor performances for smaller values of $\rho$. However, it can be seen from Figure 3 that there seem to be an exchange point in which the dominance of $\rho$ starts to increase over the thermal noise. In this point $P_{\mathrm{e}}$ achieve a minimum for a given $\rho<1$, at intermediate values of $E_{\mathrm{b}} / N_{0}$. Nonetheless, one must be aware that actual estimators typically achieve better accuracies at high signal-to-noise ratios, meaning that the inflections shown in Figure 3 might not occur, yielding a monotonically decreasing probability of error with increasing values of $E_{\mathrm{b}} / N_{0}$.

\section{E. Performance under the joint effect of all impairments}

It is not uncommon that the performance degradation is not additive when a system is subjected to the joint effect of impairments and uncertainty in several parameters at the same time. In other words, it can happen that the performance degradation produced by a given shift of an impairment or in the estimate of a parameter be somehow compensated for by a shift in another impairment or parameter estimate. To assess this situation, Figure 4 shows the global spectrum sensing performance in terms of $P_{\mathrm{e}}$ versus $E_{\mathrm{b}} / N_{0}$, as achieved by the adaptive receiver under the joint effect of uncertainty in the noise variance with $\mu_{\mathrm{dB}}=0,1,-1 \mathrm{~dB}$, and $\mu \sim$ $\mathcal{U}[1 / 1.259,1.259]$, in the report channel gain with $\rho=0.99$ 

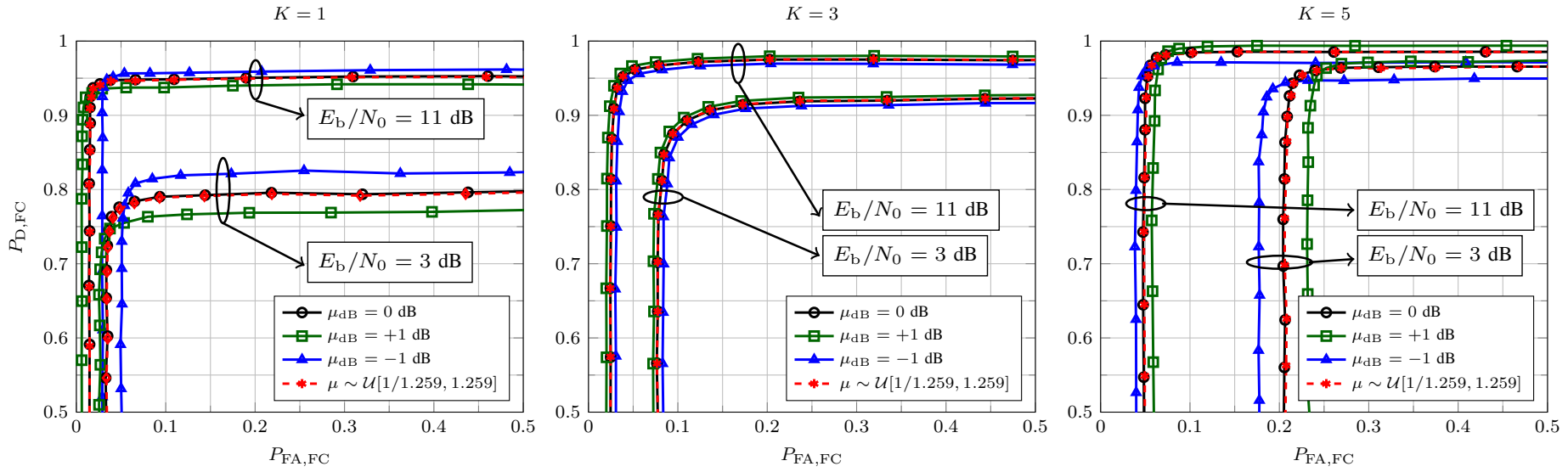

Fig. 2. Global spectrum sensing performance in terms of ROCs, as achieved by the adaptive receiver under noise variance uncertainty with $\mu_{\mathrm{dB}}=0,1,-1$ $\mathrm{dB}$, and $\mu \sim \mathcal{U}[1 / 1.259,1.259]$, for $E_{\mathrm{b}} / N_{0}=3 \mathrm{~dB}$ and $11 \mathrm{~dB}, M=5$, and $K=1$ (left), $K=3$ (middle) and $K=5$ (right). This figure is better visualized in color.
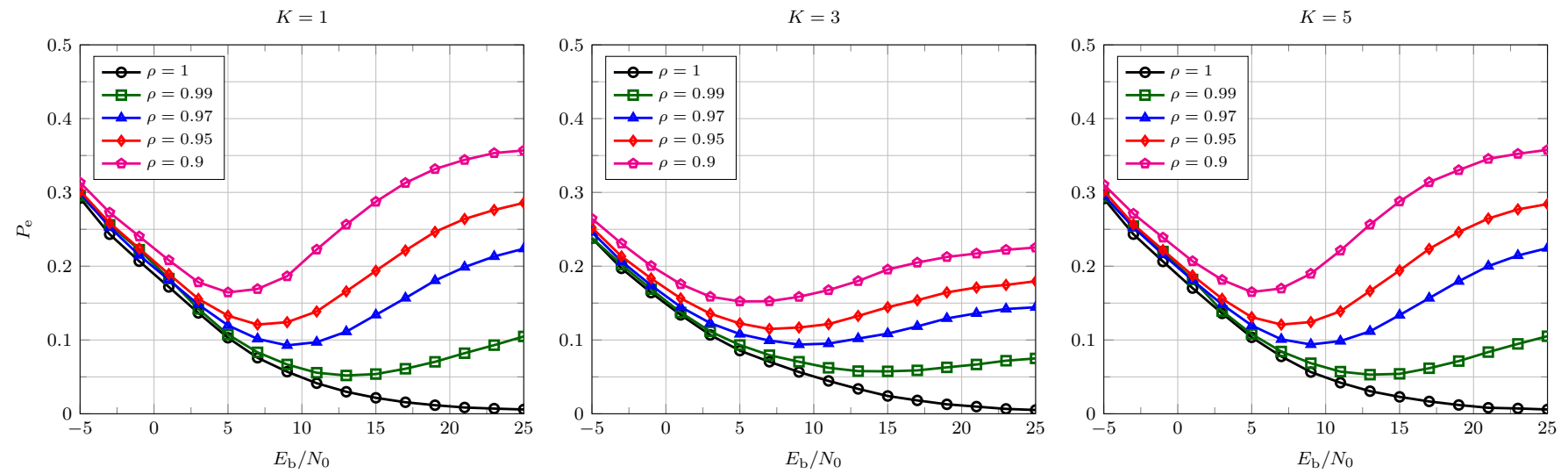

Fig. 3. Global spectrum sensing performance in terms of $P_{\mathrm{e}}$, as achieved by the adaptive receiver under uncertainty in the report channel gain with $\rho=1,0.99,0.97,0.95$ and 0.9 , as a function of $E_{\mathrm{b}} / N_{0} \mathrm{~dB}$, for $M=5$, and $K=1$ (left), $K=3$ (middle) and $K=5$ (right). This figure is better visualized in color.
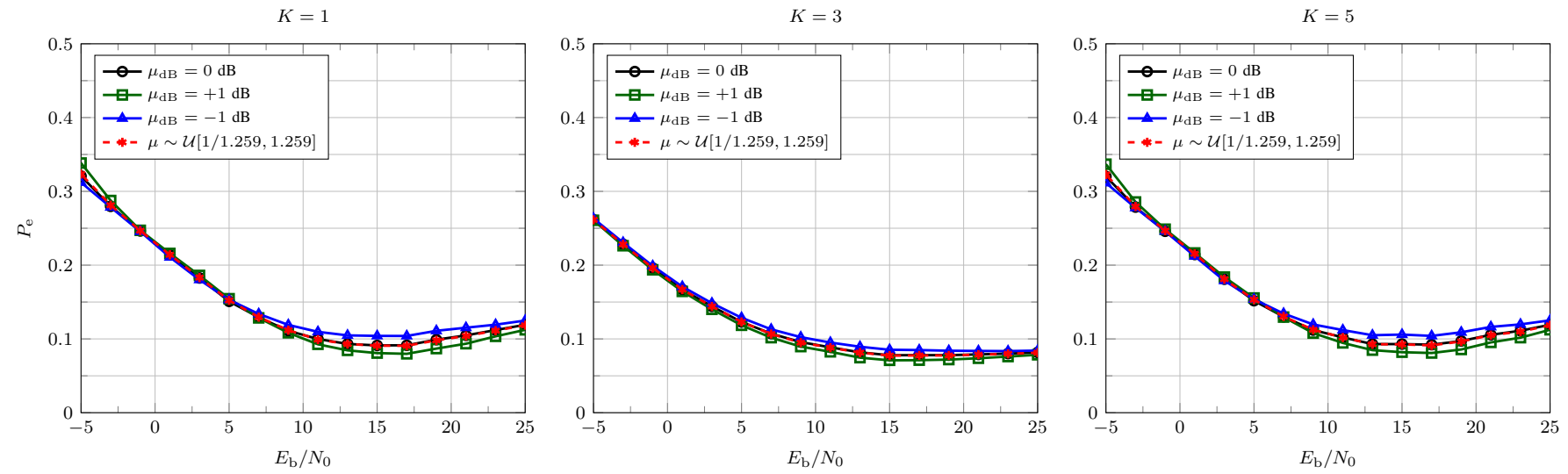

Fig. 4. Global spectrum sensing performance in terms of $P_{\mathrm{e}}$ versus $E_{\mathrm{b}} / N_{0}$, as achieved by the adaptive receiver under the joint effect of uncertainty in the noise variance with $\mu_{\mathrm{dB}}=0,1,-1 \mathrm{~dB}$, and $\mu \sim \mathcal{U}[1 / 1.259,1.259]$, in the report channel gain with $\rho=0.99$ and under impulsive noise with $\alpha=1.5$, for $M=5$, and $K=1$ (left), $K=3$ (middle) and $K=5$ (right). This figure is better visualized in color. 
and under impulsive noise with $\alpha=1.5$, for $M=5$, and $K=1,3$ and 5 .

From Figure 4 it can be seen that the performance degradation due to noise uncertainty is indeed small, as already verified from Figure 2. However, the joint effect of impulsive noise and a small channel uncertainty $(\rho=0.99)$ has prevented the system to achieve the target $P_{\mathrm{e}}$ of 0.05 , no matter the value of $E_{\mathrm{b}} / N_{0}$. Among the combining rules AND, OR and MAJ, again the MAJ is the most promising, closely approaching the target $P_{\mathrm{e}}$ for $E_{\mathrm{b}} / N_{0}>15 \mathrm{~dB}$.

\section{CONCLUSIONS}

This paper presented an analysis of the global spectrum sensing performance of a resource efficient fusion scheme when the fusion center receiver is subjected to impulsive noise and uncertainty in the channel state information and thermal noise variance. It was demonstrated that the receiver is quite robust against noise uncertainty and impulsive noise, whereas its performance may be severely degraded due to channel state information uncertainty. Specifically in the case of noise uncertainty, it has been shown that unbiased estimators of the noise variance, not necessarily accurate, will tend to keep the performance of the spectrum sensing practically unchanged with respect to the perfect knowledge of this parameter.

The results shown in this paper has unveiled a considerable weakness of the resource efficient fusion scheme based on the idea of [17]: the need of knowing the noise variance and the report channel gains, especially knowing the channel gains by means of a very accurate estimation process. The correlation coefficient between the estimated channel gains and the actual ones must be higher than 0,99 if one intends to have a monotonic increase in the system performance as the received signal-to-noise ratio per bit is increased.

It remains an open problem, and an opportunity of further contributions, the development of analytical results concerning the performance of the receiver considered in this paper under all above-mentioned impairments, or at least under the most degrading one: the channel state information error.

\section{ACKNOWLEDGMENT}

This work was partially supported by Finep, with resources from Funttel, Grant No. 01.14.0231.00, under the Radiocommunication Reference Center (Centro de Referência em Radiocomunicações, CRR) project of the National Institute of Telecommunications (Instituto Nacional de Telecomunicações, Inatel), Brazil.

\section{REFERENCES}

[1] U. S. F. C. C. S. P. T. Force, Spectrum Policy Task Force Report. Federal Communications Commission, Spectrum Policy Task Force, 2002. [Online]. Available: https://books.google.com.br/books?id=p9MQtwAACAAJ

[2] S. W. Oh, Y. Ma, M.-H. Tao, and E. Peh, Introduction to Cognitive Radio and Television White Space. Wiley-IEEE Press, 2017, pp. 360-, doi: 10.1002/9781119110491.ch1.

[3] J. Mitola and G. Q. Maguire, "Cognitive radio: making software radios more personal," IEEE Personal Communications, vol. 6, no. 4, pp. 1318, Aug. 1999, doi: 10.1109/98.788210.
[4] S. Haykin, "Cognitive radio: brain-empowered wireless communications," IEEE Journal on Selected Areas in Communications, vol. 23, no. 2, pp. 201-220, Feb. 2005, doi: 10.1109/JSAC.2004.839380.

[5] X. Hong, J. Wang, C.-X. Wang, and J. Shi, "Cognitive radio in 5G: a perspective on energy-spectral efficiency trade-off," IEEE Communications Magazine, vol. 52, no. 7, pp. 46-53, Jul. 2014, doi: 10.1109/MCOM.2014.6852082.

[6] I. F. Akyildiz, B. F. Lo, and R. Balakrishnan, "Cooperative spectrum sensing in cognitive radio networks: A survey," Else vier Physical Communications, vol. 4, pp. 40-62, Mar. 2011, doi: 10.1016/j.phycom.2010.12.003.

[7] C. Sun, W. Zhang, and K. B. Letaief, "Cluster-based cooperative spectrum sensing in cognitive radio systems," in Proc. of IEEE International Conference on Communications, Jun. 2007, pp. 2511-2515, doi: 10.1109/ICC.2007.415

[8] Q. Zou, S. Zheng, and A. H. Sayed, "Cooperative sensing via sequential detection," IEEE Transactions on Signal Processing, vol. 58, no. 12, pp. 6266-6283, Dec. 2010, doi: 10.1109/TSP.2010.2070501.

[9] H. Vu-Van and I. Koo, "A sequential cooperative spectrum sensing scheme based on cognitive user reputation," IEEE Transactions on Consumer Electronics, vol. 58, no. 4, pp. 1147-1152, Nov. 2012, doi: 10.1109/TCE.2012.6414979.

[10] C. Q. Liu and Z. M. Wang, "Adaptive weighted algorithm of cooperative spectrum sensing in cognitive radio networks," in Proc. of IET International Communication Conference on Wireless Mobile and Computing, Nov. 2011, pp. 121-126, doi: 10.1049/cp.2011.0860.

[11] Y. Zou, Y. D. Yao, and B. Zheng, "A cooperative sensing based cognitive relay transmission scheme without a dedicated sensing relay channel in cognitive radio networks," IEEE Transactions on Signal Processing, vol. 59, no. 2, pp. 854-858, Feb. 2011, doi: 10.1109/TSP.2010.2090876.

[12] —, "A selective-relay based cooperative spectrum sensing scheme without dedicated reporting channels in cognitive radio networks," IEEE Transactions on Wireless Communications, vol. 10, no. 4, pp. 11881198, Apr. 2011, doi: 10.1109/TWC.2011.021611.100913.

[13] S. Mitsuya, K. Kobayashi, T. Yamazato, and M. Katayama, "Improvement of sequential-test-based cooperative spectrum sensing systems in band limited control channels," in Proc. of $10^{\text {th }}$ International Symposium on Communications and Information Technologies, Oct. 2010, pp. 968 973, doi: 10.1109/ISCIT.2010.5665127.

[14] C. Sun, W. Zhang, and K. B. Letaief, "Cooperative spectrum sensing for cognitive radios under bandwidth constraints," in Proc. of IEEE Wireless Communications and Networking Conference, Mar. 2007, pp. 1-5, doi:10.1109/WCNC.2007.6.

[15] M. Ghaznavi and A. Jamshidi, "Efficient method for reducing the average control bits in a distributed cooperative sensing in cognitive radio system," IET Communications, vol. 7, no. 9, pp. 867-874, Jun. 2013, doi: 10.1049/iet-com.2012.0574.

[16] K. Umebayashi, J. J. Lehtomaki, T. Yazawa, and Y. Suzuki, "Efficient decision fusion for cooperative spectrum sensing based on or-rule," IEEE Transactions on Wireless Communications, vol. 11, no. 7, pp. 25852595, Jul. 2012, doi: 10.1109/TWC.2012.052412.111727.

[17] C. H. Lim, "Resource-efficient transmission for report channel in cooperative spectrum sensing," Electronics Letters, vol. 50, no. 16, pp. 1171-1173, Jul. 2014, doi: 10.1049/el.2014.1469.

[18] K. B. Letaief and W. Zhang, "Cooperative communications for cognitive radio networks," Proceedings of the IEEE, vol. 97, no. 5, pp. 878-893, May 2009, doi: 10.1109/JPROC.2009.2015716.

[19] D. A. Guimarães, G. P. Aquino, and M. E. G. V. Cattaneo, "Improved decision for a resource-efficient fusion scheme in cooperative spectrum sensing," in Proc. of International Workshop on Telecommunications, Jun. 2015, pp. 1-5, doi: 10.1109/IWT.2015.7224556.

[20] D. A. Guimarães, G. P. Aquino, and P. H. Souza, "Receptor adaptativo para fusão eficiente de decisões no sensoriamento espectral cooperativo," in XXXIV Simpósio Brasileiro de Telecomunicações e Processamento de Sinais (SBrT 2016), Santarém, Brazil, Aug. 2016.

[21] M. Gans, "The effect of gaussian error in maximal ratio combiners," IEEE Transactions on Communication Technology, vol. 19, no. 4, pp. 492-500, Aug. 1971, doi: 10.1109/TCOM.1971.1090666.

[22] R. Annavajjala and L. B. Milstein, "Performance analysis of linear diversity-combining schemes on rayleigh fading channels with binary signaling and gaussian weighting errors," IEEE Transactions on Wireless Communications, vol. 4, no. 5, pp. 2267-2278, Set. 2005, doi: 10.1109/TWC.2005.853834.

[23] R. Annavajjala, P. C. Cosman, and L. B. Milstein, "Performance analysis of linear modulation schemes with generalized diversity combining on rayleigh fading channels with noisy channel estimates," IEEE Transac- 
tions on Information Theory, vol. 53, no. 12, pp. 4701-4727, Dec. 2007, doi: 10.1109/TIT.2007.909130.

[24] H. A. Suraweera, P. J. Smith, and M. Shafi, "Capacity limits and performance analysis of cognitive radio with imperfect channel knowledge," IEEE Transactions on Vehicular Technology, vol. 59, no. 4, pp. 18111822, May 2010, doi: 10.1109/TVT.2010.2043454.

[25] M. Lopez-Benitez and F. Casadevall, "Signal uncertainty in spectrum sensing for cognitive radio," IEEE Transactions on Communications, vol. 61, no. 4, pp. 1231-1241, Apr. 2013, doi: 10.1109/TCOMM.2013.021413.110807.

[26] A. Sahai, N. Hoven, and R. Tandra, "Some fundamental limits on cognitive radio," in Proc. of $42^{\text {th }}$ Allerton Conference on Communication, Control, and Computing, 2004.

[27] D. A. Guimarães and R. A. A. de Souza, "Simple and efficient algorithm for improving the MDL estimator of the number of sources," Sensors, vol. 14 , no. 10 , p. 19477,2014 , doi: $10.3390 / \mathrm{s} 141019477$.

[28] M. Shao and C. L. Nikias, "Signal processing with fractional lower order moments: stable processes and their applications," Proceedings of the IEEE, vol. 81, no. 7, pp. 986-1010, Jul. 1993, doi: 10.1109/5.231338.

[29] G. A. Tsihrintzis and C. L. Nikias, "Performance of optimum and suboptimum receivers in the presence of impulsive noise modeled as an alpha-stable process," IEEE Transactions on Communications, vol. 43, no. 2/3/4, pp. 904-914, Feb. 1995, doi: 10.1109/26.380123.

[30] D. A. Guimarães, R. A. A. de Souza, and A. N. Barreto, "Performance of cooperative eigenvalue spectrum sensing with a realistic receiver model under impulsive noise," Journal of Sensor and Actuator Networks, vol. 2, no. 1, pp. 46-69, 2013, doi: 10.3390/jsan2010046. [Online]. Available: http://www.mdpi.com/2224-2708/2/1/46

[31] Altair Engineering, Inc. (former Visual Solutions, Inc.), "VisSim: A graphical language for simulation and model-based embedded development," Nov. 2016.

[32] D. A. Guimarães, Digital Transmission: A Simulation-Aided Introduction with VisSim/Comm. Springer-Verlag Berlin Heidelberg, 2009, doi: 10.1007/978-3-642-01359-1.

[33] L. Khalid and A. Anpalagan, "Reliability-based decision fusion scheme for cooperative spectrum sensing," IET Communications, vol. 8, no. 14, pp. 2423-2432, Sep. 2014, doi: 10.1049/iet-com.2014.0205.

[34] S. Atapattu, C. Tellambura, and H. Jiang, "Energy detection based cooperative spectrum sensing in cognitive radio networks," IEEE Transactions on Wireless Communications, vol. 10, no. 4, pp. 1232-1241, Apr. 2011, doi: 10.1109/TWC.2011.012411.100611.

[35] The Institute of Electrical and Electronic Engineers, "IEEE Standard for Information Technology-Telecommunications and information exchange between systems Wireless Regional Area Networks (WRAN)-Specific requirements Part 22: Cognitive Wireless RAN Medium Access Control (MAC) and Physical Layer (PHY) Specifications: Policies and Procedures for Operation in the TV Bands," IEEE Std 802.22-2011, pp. 1-680, Jan. 2011, doi: 10.1109/IEEESTD.2011.5951707.

[36] D. A. Guimares and G. P. Aquino, "Resource-efficient fusion over fading and non-fading reporting channels for cooperative spectrum sensing," Sensors, vol. 15, no. 1, pp. 1861-1884, Jan. 2015, doi: 10.3390/s150101861.

[37] S. Boyd and L. Vandenberghe, Convex Optimization. New York, NY, USA: Cambridge University Press, 2004.

[38] F. Bellili, R. Meftehi, S. Affes, and A. Stphenne, "Maximum likelihood SNR estimation over time-varying flat-fading SIMO channels," in Proc. of IEEE International Conference on Acoustics, Speech and Signal Processing, May 2014, pp. 6523-6527, doi: 10.1109/ICASSP.2014.6854861.

[39] J. Lago-Fernández and J. Salter, "Modeling impulsive interference in DVB-T: Statistical analysis, test waveforms and receiver performance," BBC R\&D White Paper WHP 080, Apr. 2004.

[40] D. Middleton, "Non-gaussian noise models in signal processing for telecommunications: new methods an results for class a and class b noise models," IEEE Transactions on Information Theory, vol. 45, no. 4, pp. 1129-1149, May 1999, doi: 10.1109/18.761256.

[41] I. Mann, S. McLaughlin, W. Henkel, R. Kirkby, and T. Kessler, "Impulse generation with appropriate amplitude, length, inter-arrival, and spectral characteristics," IEEE Journal on Selected Areas in Communications, vol. 20, no. 5, pp. 901-912, Jun. 2002, doi: 10.1109/JSAC.2002.1007373.

[42] M. Chitre, "Underwater acoustic communications in warm shallow water channels," Ph.D. dissertation, National University of Singapore, 2006.

[43] D. A. Guimarães, L. S. Chaves, and R. A. A. de Souza, "Snapping shrimp noise reduction using convex optimization for underwater acoustic communication in warm shallow water," in Proc. of Inter- national Telecommunications Symposium, Aug. 2014, pp. 1-5, doi: 10.1109/ITS.2014.6948044.

[44] P. H. C. Souza, "Vissim/comm simulation of the efficient fusion CSS," https://www.dropbox.com/sh/yn8mkzgbgidm605/AAB7JhuapQv9qgOo EticQBJ4a?dl=0.

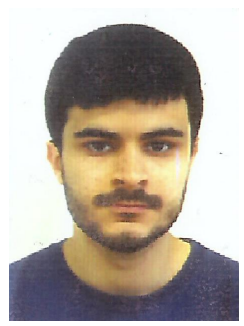

Pedro Henrique Cardoso Souza received the B.S. in electrical engineering from National Institute of Telecommunications (Inatel), Brazil, in 2015, and is currently working toward the M.Sc. degree in telecommunications engineering at Inatel. His main research interests are in digital communication systems and in cooperative spectrum sensing techniques for cognitive radio applications.

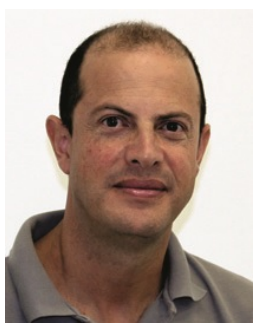

Dayan Adionel Guimarães has a $\mathrm{PhD}$ in Electrical Engineering from State University of Campinas (Unicamp), Brazil, and is currently a lecturer and researcher at the National Institute of Telecommunications (Inatel), Brazil. His research interests lie in the the general aspects of digital transmission and mobile communications. He is currently working on spectrum sensing techniques for cognitive radio applications, multi-slope propagation models and applications of convex optimization in communications.

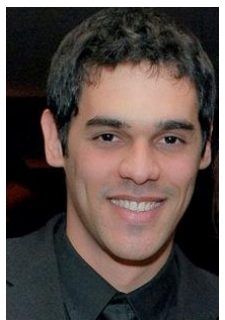

Guilherme Pedro Aquino received the M.Sc. degree in telecommunications engineering from Instituto Nacional de Telecomunicações (Inatel), Brazil, and currently is pursuing this doctorate in Electrical Engineering from Universidade Federal de Itajubá, UNIFEI, Brazil. Since 2010 he is a professor and researcher at Inatel. His main research interests lie in the study of the non-ortogonal multiple access schemes and cooperative spectrum sensing techniques for cognitive radio applications. 